\title{
Analysis of Supply and Ground Noise Sensitivity in Ring and LC Oscillators
}

\author{
Volodymyr Kratyuk, Igor Vytyaz, Un-Ku Moon, Kartikeya Mayaram \\ School of Electrical Engineering \& Computer Science \\ Oregon State University, Corvallis, OR 97331, USA
}

\begin{abstract}
Supply and ground noise sensitivity of a wide variety of ring and LC oscillators has been analyzed based on the perturbation projection vector (PPV) technique. The resulting PPV provides an understanding of how specific frequency content of supply/ground noise is converted to oscillator phase noise. Based on this analysis oscillators that are tolerant to supply/ground noise can be identified and used for low noise oscillator design.
\end{abstract}

Keywords: supply noise, ground noise, oscillators, noise in oscillators, noise analysis, perturbation projection vector.

\section{INTRODUCTION}

Phase-locked loops (PLLs) are key building blocks of frequency synthesizers and clock generators and they are used in nearly all analog, digital, and RF ICs. Within the PLL the voltage controlled oscillator (VCO) is a critical block since its noise performance determines the performance of the overall PLL. Given the drive for high levels of integration in current and future generations of integrated systems-on-a-chip (SoC), the design of VCOs is critical to ensuring first-pass silicon of future SoCs. The major components that contribute noise in VCOs are the device thermal and flicker noise, substrate coupling noise, and noise coupling through the power supply [1]. Although techniques are available for analyzing the phase noise of an oscillator due to the intrinsic thermal and flicker noise sources [2, 3], analysis of supply and substrate noise has been very limited $[4,5]$.

In this paper, we use our version of SPICE3 with the perturbation projection vector (PPV) based noise analysis technique [3] to evaluate the supply noise sensitivity of a wide range of ring and LC oscillators. The paper is organized as follows. In Section II, the PPV based noise analysis method is briefly described. Simulations of the PPV for a wide range of oscillators are presented in Section III followed by a discussion of the various structures in Section IV. Finally conclusions are provided in Section V.

\section{SIMULATION ENVIRONMENT}

For the accurate simulation of phase noise in VCOs, the circuit simulator SPICE3 has been extended to handle phase noise analysis [6] based on a non-linear perturbation analysis for oscillators [3, 7, 8]. The implemented technique allows for an accurate simulation of phase noise.

For $0 \leq f_{m}<<f_{0}$, the single-sideband phase noise spectrum $L\left(f_{m}\right)$ in $d B c / H z$ can be approximated as [8]:

$$
L\left(f_{m}\right)=10 \log _{10}\left(\frac{f_{0}^{2} c\left(f_{m}\right)}{\pi^{2} f_{0}^{4} c^{2}\left(f_{m}\right)+f_{m}^{2}}\right)
$$

where $f_{0}$ is the frequency of oscillation, $f_{m}$ is the offset frequency and the scalar constant $c\left(f_{m}\right)$ is frequency dependent in the general case and is given by:

$$
c(f)=c_{w}+\sum_{m=1}^{M}\left|c_{c m}(f)\right|^{2}
$$

where $c_{w}$ is a contribution to the scalar $c$ from white noise sources and is given by:

$$
c_{w}=\frac{1}{T} \int_{0}^{T} v_{1}^{T}(\tau) B_{w}\left(x_{s}(\tau)\right) B_{w}^{T}\left(x_{s}(\tau)\right) v_{1}(\tau) d \tau
$$

and $c_{c m}$, is the contribution to the scalar $c$ from the $m$-th colored noise source and is given by:

$$
c_{c m}=\frac{1}{T} \int_{0}^{T} v_{1}^{T}(\tau) B_{c m}\left(x_{s}(\tau)\right) d \tau
$$

where $v_{l}(\tau)$ is the perturbation projection vector (PPV) [8]. The PPV is a periodic vector which serves as a transfer function from the noise sources to the scalar $c$, and hence to the overall phase noise power spectral density. The PPV scales the amount of noise transferred to the scalar $c$ at each point of time. $B_{w}$ is a statedependent matrix that maps white noise sources with unity PSD to the system of differential algebraic equations (DAEs) which describe a circuit. $B_{c m}$ is a statedependent vector that maps the $m$-th colored noise source to the system of DAEs. Thus, in order to obtain the single-sideband phase noise spectrum $L\left(f_{m}\right)$ in $d B c / H z$, first a scalar $c$ (Eq. (2)) needs to be calculated using (3) and (4) followed by computation of the phase noise spectrum using (1). The method to obtain the PPV and matrices $B_{w}$ and $B_{c m}$ is described in $[6,8]$. The PPVs can 
be calculated based on a time-domain periodic steadystate analysis, or using the frequency-domain harmonic balance method. In this paper, both approaches have been used and the resulting PPVs are in good agreement.

Since the PPV serves as a transfer function from a noise source to the scalar $c$, it is similar to the use of the impulse sensitivity function (ISF) $[1,2]$ and can be used to identify the sensitivity of a node to noise. For this reason, one can compare the PPVs from the supply/ground for two different oscillators and evaluate their sensitivity to supply/ground noise. The oscillator with a higher magnitude for the PPV will be more sensitive to supply/ground noise. In the next section we compare a variety of ring and LC oscillators for supply/ground noise tolerance based on the PPV analysis.

\section{PPV SIMULATION RESULTS}

All the oscillators in this section were designed for an oscillation frequency of approximately $1 \mathrm{GHz}$ in a 0.18 $\mu \mathrm{m}$ CMOS process. BSIM3 MOSFET models were used for the transistor models.

\section{A. Single-ended Ring Oscillators}

Consider a three-stage ring oscillator with a simple inverter delay cell and a current starved delay cell as shown in Fig. 1. The PPVs for these two types of oscillator cells are shown in this figure as well where it is seen that the simple delay cell is less sensitive to both supply and ground noise. Also note that the PPV has a dc component as well as a $3^{\text {rd }}$ harmonic content due to the switching nature of the three stage single-ended oscillator. Even though there are six switching/inverting occurrences in a period of this oscillator, only three of them are switched to supply and the other three switch to ground, resulting in a $3^{\text {rd }}$ harmonic PPV content. For an $\mathrm{N}$-stage single-ended oscillator one will see $\mathrm{N}^{\text {th }}$ harmonic content.

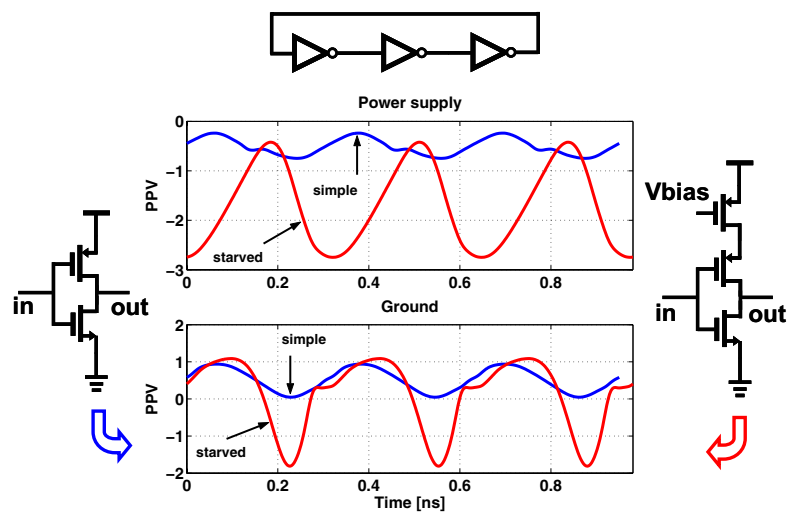

Figure 1: PPV (center) for three-stage single-ended ring oscillator with simple inverter delay cell (left) and current starved delay cell (right).

\section{B. Differential Ring Oscillators}

We consider three different ring oscillator delay cells and analyze the supply/ground noise sensitivity for a three-stage differential ring oscillator.

First we consider two commonly used delay cells: the Maneatis delay cell [9] and the Lee-Kim delay cell [10]. The PPV plots for these oscillator cells are shown in Figs. 2 and 3. From these figures one can conclude that the Lee-Kim cell is more sensitive to ground noise compared with the Maneatis delay cell in agreement with previous results [11]. Another observation is that the PPV has a dc component as well as a $6^{\text {th }}$ harmonic content due to the differential switching of the circuit. Differential switching creates paths to both supply and ground at each of the switching/inverting occurrences, resulting in $2 * \mathrm{~N}^{\text {th }}$ harmonic content in the PPV (twice the harmonic in comparison to the singled-ended case) for an $\mathrm{N}$-stage oscillator.

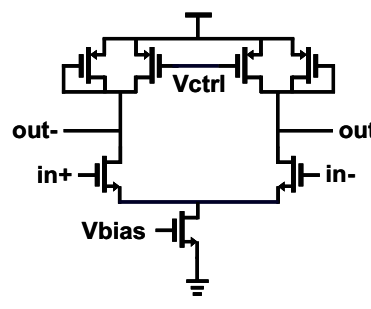

(a)

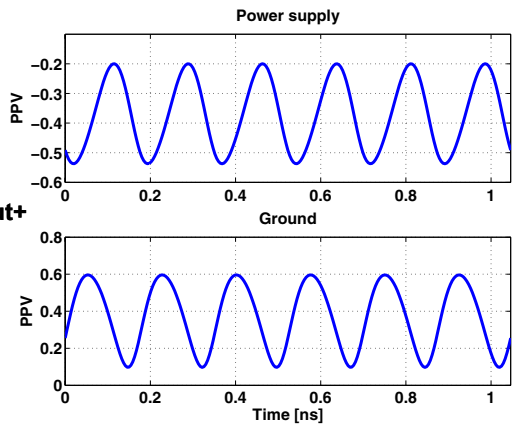

(b)
Figure 2: (a) Maneatis delay cell of [9], (b) power supply PPV (top) and ground PPV (bottom).

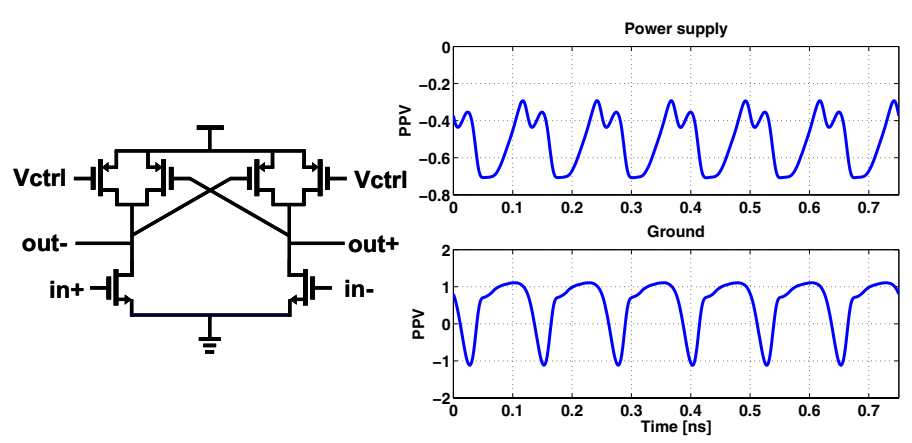

(a)

(b)

10], (b) power supply PPV (top) and ground PPV (bottom).

Next we consider the oscillator based on the delay cell shown in Fig. 4 (a) [12] and the PPV for the power supply and ground are shown in Fig. 4(b). There are two different PPV analyses shown in Fig. 4(b) one with an ideal ground connection (short) and one with a small resistance (R). The PPV analysis provides insight into the 
noise sensitivity of the supply and ground nodes. By comparing the PPV magnitudes we see that the delay cell with an ideal ground connection is more sensitive to ground noise and not as sensitive to power supply noise. The current sources at the top make this cell more tolerant to supply noise. For this reason we expect that the delay cell with a small resistance to ground would provide an improved noise immunity. This result is observed in Fig. 4(b) where the ground PPV reduces in magnitude. However, an adverse affect is observed on the supply as that PPV increases in magnitude.

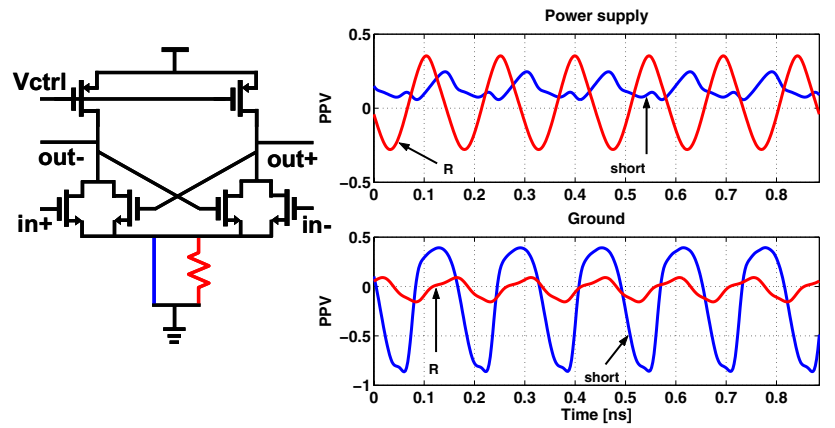

(a)

(b)

Figure 4: (a) Delay cell of [12], (b) power supply PPV (top) and ground PPV (bottom).

The oscillators analyzed above and the resulting PPV provides additional insight. For example, when the PPV results in a dc component plus a $6^{\text {th }}$ harmonic, it indicates an up-conversion of dc (low frequency) supply noise to the carrier's phase noise as well as down-conversion of supply noise at the $6^{\text {th }}$ harmonic frequency to the carrier's phase noise. This insight provides the designer some control in choosing the number of stages (which dictates the harmonics of the PPV) used in the oscillator if reasonable information about the supply/ground noise spectral content is available.

\section{Single-ended LC (Colpitts) Oscillator}

A simple Colpitts oscillator is included here as an example of a single-ended LC oscillator. The circuit and the PPV for supply and ground nodes are shown in Figs.
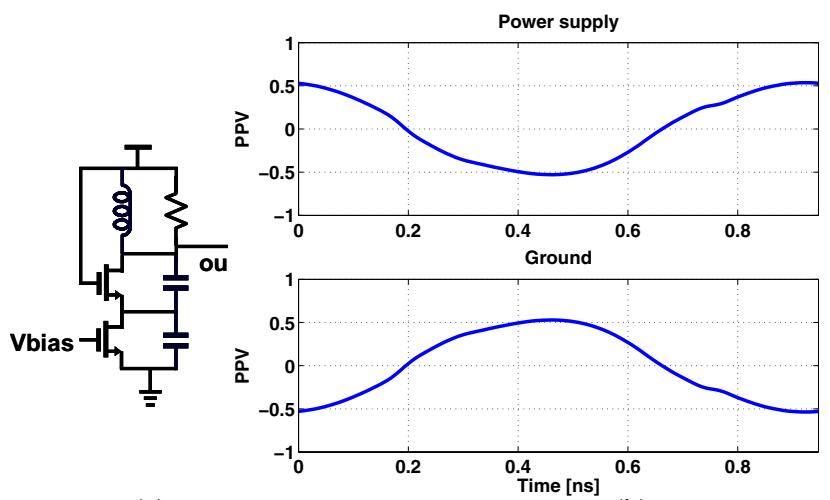

(a)

(b)

Figure 5: (a) Colpitts oscillator, (b) power supply PPV (top) and ground PPV (bottom). 5(a) and 5(b), respectively. From the PPV we see that this circuit has a similar sensitivity to supply and ground noise since the PPVs are identical in magnitude. One difference compared with the single-ended ring oscillator is the absence of the $3^{\text {rd }}$ harmonic in the PPV. This is because there is no switching of individual stages as in the 3 stage ring oscillator. So called "switching" occurs only once to supply and once to ground in a period of oscillation.

The PPVs for a NMOS cross-coupled oscillator (Fig. 6(a)) are shown in Fig. 6(b). In this case we see that the PPVs for both the supply and ground are of similar magnitude. The PPVs display a $2^{\text {nd }}$ harmonic (and $4^{\text {th }}, 6^{\text {th }}$, etc.) due to the fact that the differential LC oscillator "switches" to both supply and ground two times per period of oscillation.

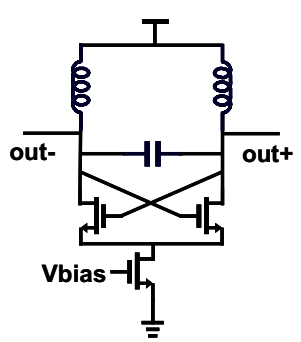

(a)
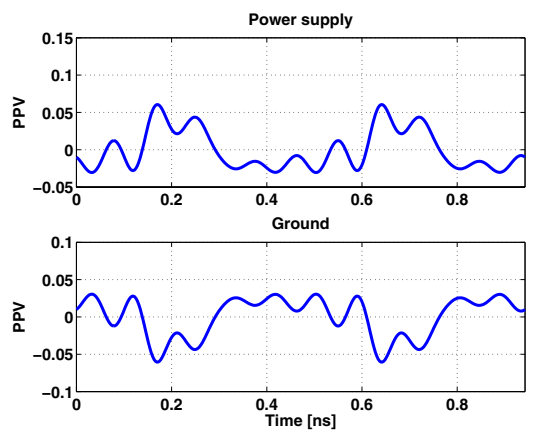

(b)

Figure 6: (a) NMOS cross-coupled oscillator, (b) power supply PPV (top) and ground PPV (bottom).

A complementary cross-coupled oscillator of Fig. 7(a) is analyzed with different options, such as a NMOS tail current source before the ground connection (tail CS), a small resistance (R), and an LC filter that is tuned to the second harmonic of the oscillation frequency (LC). PPVs for these cases together with PPVs of the oscillator with no modifications (short) are shown in Fig. 7(b). From this figure we see that a direct ground connection results in the most sensitivity to supply/ground noise. The use of a tail current source, resistor, or the LC filter are effective techniques to reduce the magnitude of the PPV and hence the sensitivity to supply/ground noise. It is interesting to note that when an LC tank isolation is used to suppress the down-conversion of supply/ground noise at the $2^{\text {nd }}$ harmonic, the PPV changes from having the $2^{\text {nd }}$ to $4^{\text {th }}$ harmonic component. While one normally does not have the choice to change the number of stages in the design of an LC oscillator, such a supply/ground filtering technique effectively changes the PPV characteristic that can represent a multi-stage oscillator behavior. Thus a similar design flexibility for LC oscillators (with respect to supply sensitivity) is achieved as in the case of ring oscillators. 


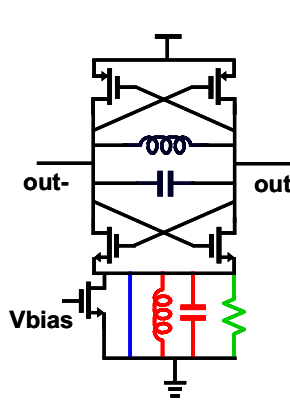

(a)

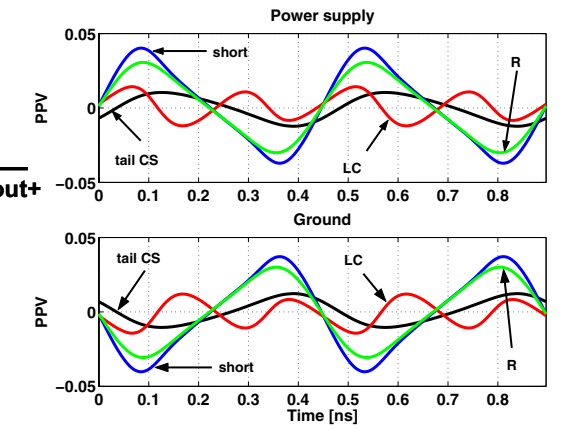

(b)
Figure 7: (a) Complementary cross-coupled oscillator, (b) power supply PPV (top) and ground PPV (bottom).

The complementary cross-coupled oscillator cell can be further modified to use resistors or LC filters in both the supply and ground connections. This modified oscillator is shown in Fig. 8(a) and the corresponding PPVs are shown in Fig. 8(b). Once again we see that the LC filter and the resistors are effective in reducing the PPV magnitude for supply and ground as in the previous design.

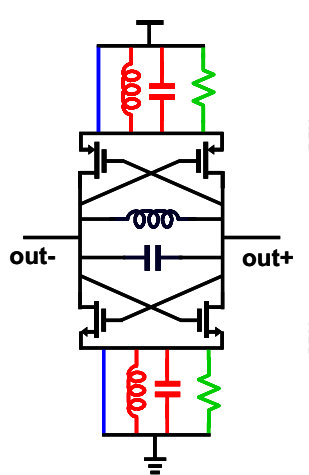

(a)
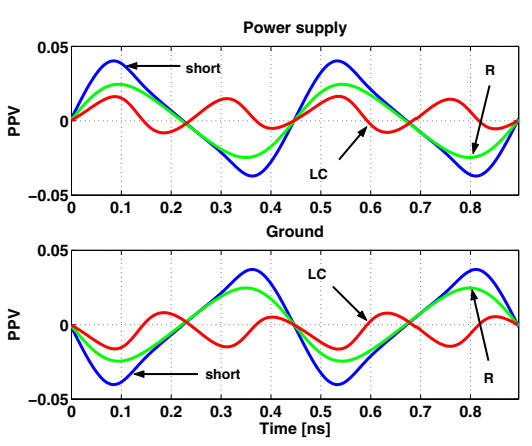

(b)

Figure 8: (a) Complementary cross-coupled oscillator, (b) power supply PPV (top) and ground PPV (bottom).

\section{DISCUSSION}

From the various simulation results the following conclusions can be derived. For ring VCOs with single ended delay cells, the phase noise is affected by supply and ground noise at baseband and (\# of stages) ${ }^{*} \mathrm{~N}$ harmonics. In the differential ring oscillators the phase noise is affected by supply and ground noise at baseband and $2 *$ (\# of stages) $* \mathrm{~N}$ harmonics. In a single-ended LC oscillator, the phase noise is affected by supply and ground noise at the baseband and harmonics of the oscillation frequency. However, for a differential LC oscillator, supply and ground noise at the baseband and even harmonics of the oscillation frequency are important. Furthermore, use of LC tanks to isolate supply/ground noise in LC oscillators results in the modification of PPVs in a similar manner to that for ring oscillators with different number of stages.

\section{CONCLUSION}

A simulation technique based on the perturbation projection vector (PPV) has been used to accurately simulate the supply/ground noise sensitivity of a wide variety of ring and LC oscillators. Based on this analysis insight is gained into the supply/ground noise sensitivity of the various oscillators. This work provides the foundation for identifying oscillators that are tolerant to supply and ground noise.

\section{ACKNOWLEDGMENTS}

This work is supported in part by the SRC under contracts 2003-HJ-1076 and 2001-TJ-922, and by NSF under grant CCR-0120275.

\section{REFERENCES}

[1] A. Hajimiri and T. H. Lee, The Design of Low Noise Oscillators, Kluwer Academic Publishers, Boston, 2000.

[2] A. Hajimiri and T. Lee, "A general theory of phase noise in electrical oscillators," IEEE J. Solid-State Circuits, vol. 33, pp. 179-194, Feb. 1998.

[3] A. Demir, A. Mehrotra, and J. Roychowdhury, "Phase noise in oscillators: a unifying theory and numerical methods for characterization," IEEE Trans. Circuit Syst.-I, vol. 47, pp. 655-674, May 2000.

[4] F. Herzel and B. Razavi, "A study of oscillator jitter due to supply and substrate noise," IEEE Trans. Circuits Syst.-II, vol. 46, pp. 56-62, Jan. 1999.

[5] N. Barton, D. Ozis, T. Fiez, and K. Mayaram, "The effect of supply and substrate noise on jitter in ring oscillators," CICC'02, pp. 505-508, May 2002.

[6] V. Kratyuk, "Algorithms and tools for optimization of integrated RF VCOs," M.S. Thesis, Oregon State University, June 2003.

[7] A. Demir, "Phase noise in oscillators: DAEs and colored noise sources," in Proc. ICCAD, pp. 170-177, Nov. 1998.

[8] A. Demir, "Floquent theory and non-linear perturbation analysis for oscillators with differential-algebraic equations," International Journal of Circuit Theory and Applications, pp. 163-185, March-April 2000.

[9] J. Maneatis and M. Horowitz, "Precise delay generation using coupled oscillators," IEEE J. Solid-State Circuits, vol. 28, pp. 1273-1282, Dec. 1993.

[10] J. Lee and B. Kim, "A low-noise fast-lock phase-looked loop with adaptive bandwidth control," IEEE J. Solid-State Circuits, vol. 35, pp. 1137-1145, 2000.

[11] M. Brownlee, P. K. Hanumolu, U. Moon, and K. Mayaram, "The effect of power supply noise on ring oscillator phase noise," Proc. NEWCAS, pp. 225-228, June 2004.

[12] R. Prasun, "A 0.6-1.2V low-power configurable PLL architecture for $6 \mathrm{GHz}-300 \mathrm{MHz}$ applications in a $90 \mathrm{~nm}$ CMOS process," Symposium on VLSI circuits, pp. 232-235, June 2004. 